\title{
MAGNETIC ERROR ANALYSIS OF RECYCLER PBAR INJECTION TRANSFER LINE
}

\author{
M.J. Yang ${ }^{\ddagger}$, Fermilab $^{\dagger}$, Batavia, IL 60510 , USA
}

\begin{abstract}
Detailed study of Fermilab Recycler Ring anti-proton injection line became feasible with its BPM system upgrade, though the beamline has been in existence and operational since year 2000. Previous attempts were not fruitful due to limitations in the BPM system. Among the objectives are the assessment of beamline optics and the presence of error fields. In particular the field region of the permanent Lambertson magnets at both ends of R22 transfer line will be scrutinized.
\end{abstract}

\section{INTRODUCTION}

The Fermilab Recycler Ring (RR) was built entirely with permanent magnets except for the correction elements. Combined function magnets, with and without sextupole field, and quadrupole magnets were used for the entire ring and its associated transfer lines. Permanent magnet Lambertsons were also used at all injection and extraction points, as shown in Figure 1. R22 line, between RR 214 to Main Injector (MI) 222 location, is used for anti-proton injection and proton extraction. R32 line, between RR 328 and MI 321 location, is for transfer in the other direction. The abort line, starting with LAM402, takes proton beams to the dump.

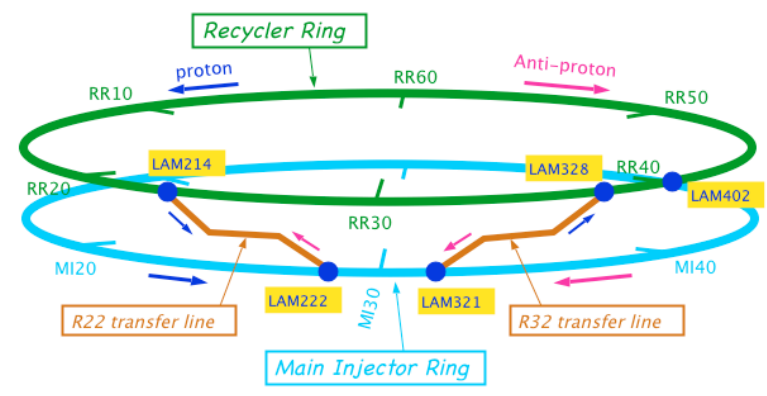

Figure 1. Diagram of Recycler Ring, Main Injector, and the two transfer lines in between. Proton beams run counterclockwise while anti-protons run clockwise. Blue dots are where permanent Lambertson magnets are located.

During commissioning of the Recycler Ring studies were conducted exclusively with protons through the R32 transfer line. It was realized at the time that substantial error fields existed in both LAM328 at Recycler end and LAM321 at Main Injector end. With limited BPM precision rough estimates of skew quadrupole components were made and corrections were installed at both locations using permanent skew quadrupoles.

The study of the R22 line, following the Recycler BPM system upgrade and that of Main Injector, is expected to

$\dagger$ Operated by Fermi Research Alliance, LLC under Contract No. DEAC02-07CH1 1359 with the United States Department of Energy.

* E-mail: yang@fnal.gov provide better understanding of transfer line optics and error fields, particularly that in LAM214 of Recycler and in LAM222 at the other end of R22 transfer line.

Data taken during study were analyzed using the technique of harmonic decomposition, of which the concept has been reported [1]. Previous application of harmonic decomposition dealt only with error fields in field-free region of Lambertson magnets of Recycler Ring [2], with LAM214 included. The analysis presented in this paper, on the other hand, will focus on the field region of the magnet. Linear beamline optical property of the transfer line will also be compared with model calculation.

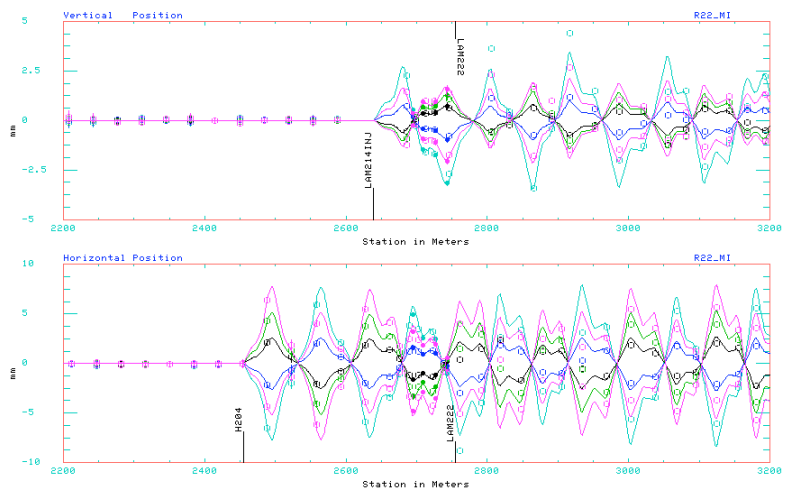

Figure 2. Orbit oscillation excited by horizontal corrector H204 in Recycler when proton beam is extracted to Main Injector. Data with six different current settings in H204 are displayed, with horizontal plane at the bottom and vertical plane on top. Results of model calculation based on corrector currents are shown in traces of corresponding color.

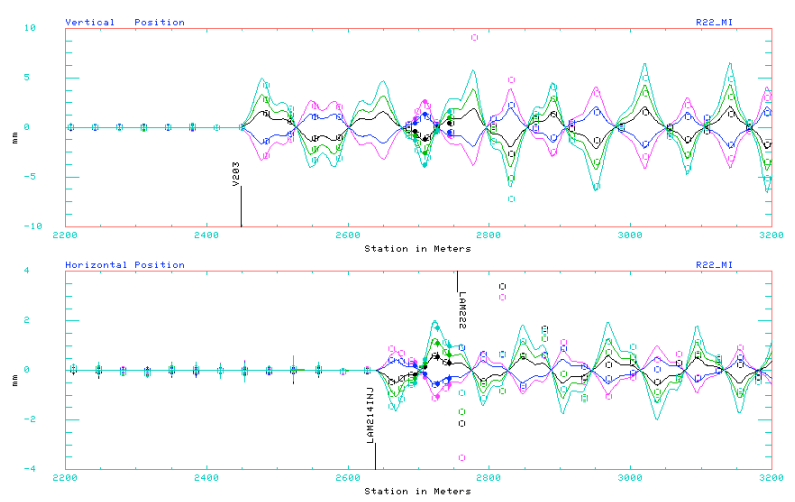

Figure 3. Orbit oscillation excited by vertical corrector V203 at five different current settings.

\section{DATA}

Two horizontal plane and two vertical plane correctors were used to excite orbit oscillation. These correctors are located within Recycler Ring, just before extraction point. Separation in phase advances between same plane correctors is close to $90^{\circ}$. One-bump data sets with each corrector at multiple settings were taken for analysis. Figure 2 shows one set of such data with H204 corrector. Included 
are that of Recycler last turn, R22 line, and Main Injector first turn. Data set with vertical corrector V203 is shown in Figure 3, with 5 different current settings.

In order to facilitate single dipole kick to the extracted beam while beam is circulating, extended closed orbit bumps are constructed and used. Each of these bumps starts with the intended corrector and ends after the extraction Lambertson magnet LAM214. This ensures that beam positions before the corrector remained fixed while the kick size is varied. How well the bumps worked is demonstrated in bottom plot of Figure 2 and in top plot of Figure 3, where the positions before corrector were essentially unchanged. On the other hand, with closed bump ending after extraction Lambertson magnet, the extracted beam saw no correctors to close the bump and continued to oscillation, generating single bump orbit as intended.

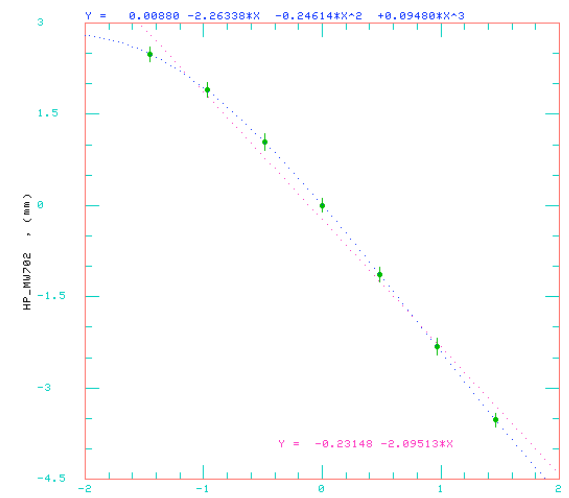

Figure 4. Horizontal position at MW702, a profile monitor, as a function of horizontal corrector H204 current. Linear fit is shown as dotted magenta line and second order fit the dotted blue line. The non-linear nature of the data is obvious.

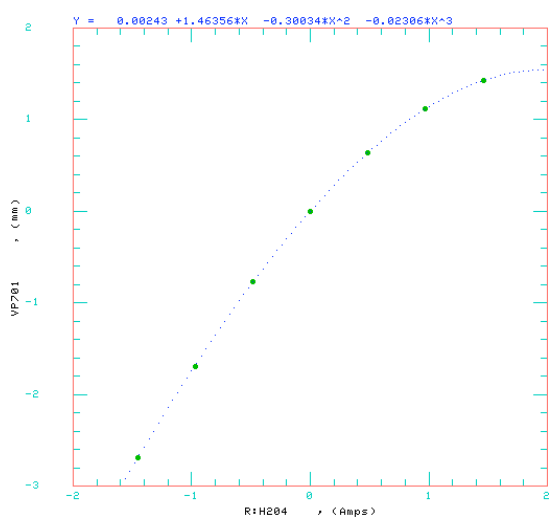

Figure 5. Vertical position at VP701, as a function of horizontal corrector H204, exhibiting signs of cross-plane coupling.

\section{ANALYSIS}

With harmonic decomposition each data set, i.e. one corrector at various kick sizes, is turned into harmonic orbits of first, second, and possibly higher orders. The first step of decomposition is demonstrated in Figure 4, where position data at MW702 is plotted against the current used in the horizontal corrector H204. Linear and second order fits are both shown to highlight the non-linear nature of position response and the need for harmonic decomposition. Shown in Figure 5 is vertical position at BPM
VP701 as a function of same corrector H204, an indication of non-linear cross-plane coupling. The coefficients of fitted polynomial, listed at top of plot, are used as magnitudes of harmonic orbits of corresponding order.

Once constructed, harmonic orbits propagates in linear optics only. BPM resolution and beam stability limits the number of orders that can be realistically analyzed. Dipole errors are always compensated by beamline correctors through orbit smoothing program and will not be the subject of discussion here.

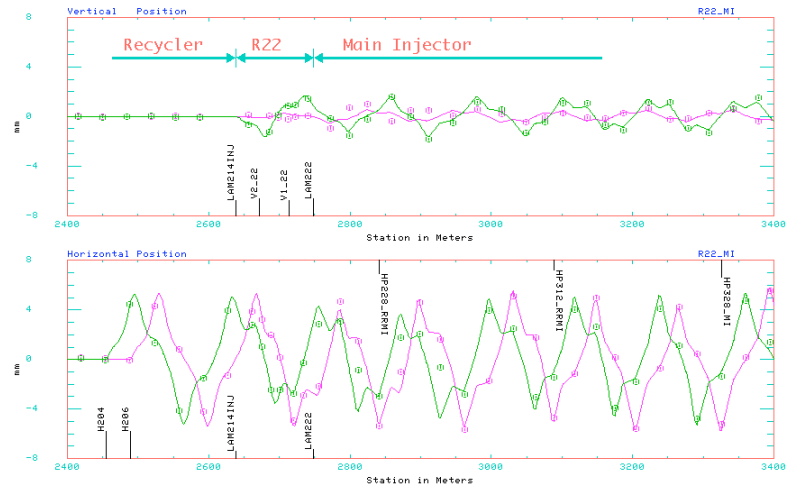

Figure 6. First order harmonic orbit from Recycler Ring to Main Injector. The data from horizontal corrector H204 is plotted in green and data from H206 in magenta.

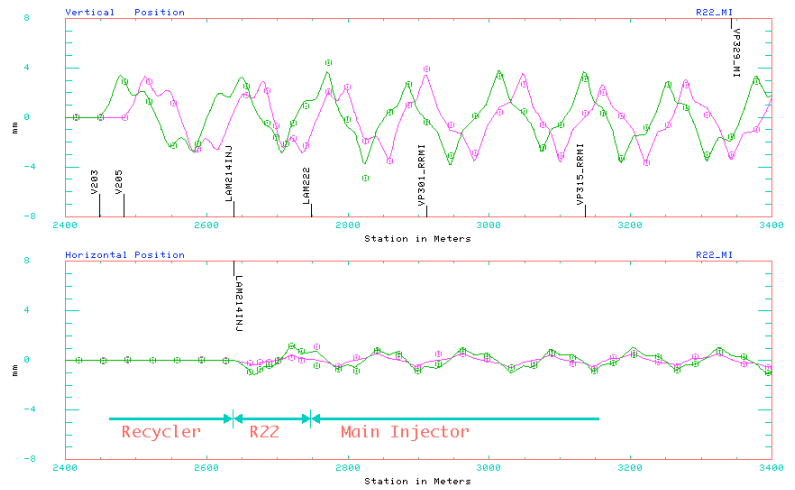

Figure 7. First order harmonic orbit from Recycler Ring to Main Injector from vertical corrector from V203 in green and from V205 in magenta.

\section{First order}

Figure 6 shows the first order harmonic orbits of corrector H204 in green and of H206 in magenta. In the absence of errors, the first order harmonic orbit is the same as the orbit caused by the corrector at one amp, with no coupling into the vertical plane. Any horizontal plane deviation from the model would indicate a focusing error and any non-zero vertical harmonic orbit would indicate linear coupling. The Model calculation results, with estimated quadrupole errors in LAM214 and LAM222 included to match first order harmonic orbit, are shown in traces of corresponding color. The errors included are a $1.0 \mathrm{KG} / \mathrm{M}$ quadrupole field with $-35^{\circ}$ roll in LAM214 and a 0.3 $\mathrm{KG} / \mathrm{M}$ quadrupole field with $40^{\circ}$ roll in LAM222. First order harmonic orbits for vertical correctors V203 and V205 are shown in Figure 7. 


\section{Second order orbits}

Second order harmonic orbits from $\mathrm{H} 204$ are shown in Figure 8. In vertical plane, top plot, kick errors at RR 214 and R22 705 locations were found to account for the observed orbit, as indicated by markers. In the horizontal plane errors were found at 212,214, and 706 location of R22 line. These kicks imply existence of second order errors. The continuation of the R22 line into the Main Injector appeared to indicate yet another kick error. However, data from MI segment seemed less reliable than expected. Given that the ability of MI BPM system on beam with $2.5 \mathrm{MHz}$ RF structure has just been commissioned, more time is needed to optimize the system before data can be reliably used for analysis.

For data with the other horizontal corrector H206, only the vertical plane harmonic orbit is shown in Figure 9. Second order kick error was found at v2_22 and v1_22, both vertical bend in R22 transfer line. Second order harmonic orbits from vertical correctors also bear signatures of errors but will not be shown.

\section{Higher order and Others}

Third and higher order errors are not supported by the available data and will not be discussed.

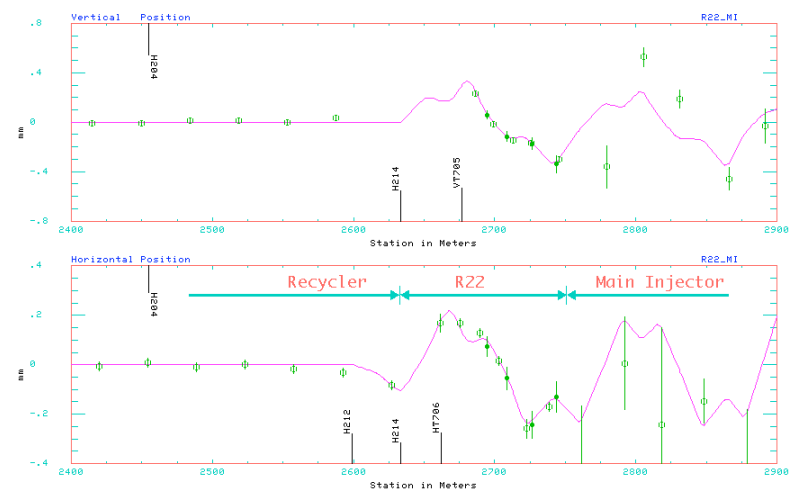

Figure 8. Second order harmonic orbit of horizontal corrector H204. Bottom plot is for horizontal plane and top plot for vertical orbit. The unit for vertical scale should be $m m / A m p^{2}$ instead.

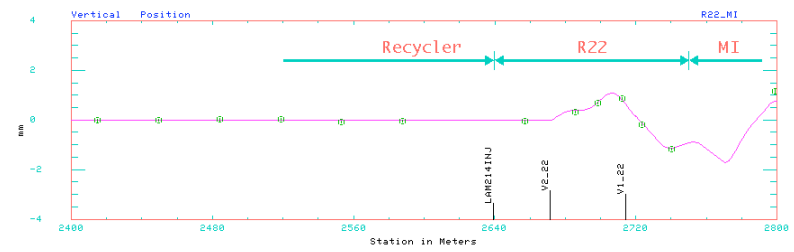

Figure 9. Second order vertical harmonic orbit from horizontal corrector H206.

\section{ERROR FIELDS}

\section{LAM214}

Of four data sets taken, only the one with H204 is most optimal for diagnosing field error at LAM214. Error multipole strengths can be calculated by interpreting kick errors found in the harmonic orbits. Table I lists the angular errors found in column two, in terms of currents in corrector H214 located next to LAM214. The field needed to cause each kick error is calculated and listed in column three. Actual multipole strengths are listed in column four. The strength of the normal quadrupole component in LAM214 was determined directly by matching to the first order orbit and used in the linear optics model for calculation.

Table I Estimated field error in LAM214 Lambertson magnet.

\begin{tabular}{|l|l|l|l|l|}
\hline & $\begin{array}{l}\text { H214 } \\
\text { amp }\end{array}$ & $\begin{array}{l}\text { Field } \\
\text { Gauss }\end{array}$ & $\begin{array}{l}\text { Pole } \\
\text { strength }\end{array}$ & unit \\
\hline N. Quad. & & & 0.8 & $K G / M$ \\
\hline S. Quad. & -0.5 & -17.4 & -0.94 & $K G / M$ \\
\hline N. Sext. & 0.04 & 1.39 & 29.8 & $K G / M^{2}$ \\
\hline S. Sext. & 0.095 & 3.3 & 70.7 & $K G / M^{2}$ \\
\hline
\end{tabular}

\section{Other error locations}

It is clear from the analysis that LAM214 is not the only element with substantial errors. The original intent was to diagnose field errors at other locations as well, including LAM222 at the Main Injector end of R22 line. The reality, however, is that down-stream analysis is very dependent on up-stream uncertainties and, in this case, there were lots of them. New data with fewer intervening field errors between corrector and target of analysis are needed to substantially improve accuracy of analysis results.

\section{CONCLUSION}

Linear optics of Recycler R22 transfer line has been established, with focusing and skew coupling error sources. Base on data analyzed, injection Lambertson magnet LAM214 was found to have substantial amount of linear and non-linear field errors and their strengths were documented. Errors in Lambertson magnet at the other end of injection transfer line, i.e. LAM222 on Main Injector, was un-mistakable. However, quantifying the error fields, and that of other locations, will take more studies that target specifically the particular location in question.

Since the study was conducted with a proton beam, the down-stream machine's ability to respond to beam with 2.5 MHz RF structure from Recycler is critical. For LAM214 down-stream machine is R22 line and data is not an issue. For LAM222 Main Injector is the downstream machine. the response of its BPM system is still to be understood.

\section{ACKNOWLEDGEMENT}

The authors would like to thank the Recycler Department for making the study time available and M. Xiao, in particular, for helping with the study. Appreciation is also given to Instrumentation department which is responsible for Recycler Ring BPM system upgrade, and made the analysis possible.

\section{REFERENCES}

[1] M.J. Yang, "Harmonic Decomposition of Orbit Data for Multipole Analysis", PAC 2005, pp. 4120-4122.

[2] M.J. Yang, M. Xiao, "Multipole Error Analysis using Local 3-Bump Orbit Data in Fermilab Recycler", PAC 2005, pp. 4144-4146. 